\title{
Prognosis of radiotherapy in medullary thyroid carcinoma patients without distant metastasis
}

\author{
Shaojun Huang", Jiana Zhong", Zhen Zhang, Rongping Chen, Jitong Li, Jia Sun, Hong Chen \\ Department of Endocrinology, Zhujiang Hospital, Southern Medical University, Guangzhou, China \\ Contributions: (I) Conception and design: S Huang, J Zhong; (II) Administrative support: H Chen; (III) Provision of study materials or patients: Z \\ Zhang; (IV) Collection and assembly of data: R Chen, J Li; (V) Data analysis and interpretation: S Huang, J Zhong, J Sun; (VI) Manuscript writing: \\ All authors; (VII) Final approval of manuscript: All authors. \\ \#These authors contributed equally to this work. \\ Correspondence to: Hong Chen; Jia Sun. No. 253, Industrial Avenue Middle, Haizhu District, Guangzhou 510282, China. \\ Email: chenhong123@smu.edu.cn; sunjia@smu.edu.cn.
}

Background: Medullary thyroid carcinoma (MTC) is an advanced disease with a poor prognosis. Although radiotherapy is widely utilized to treat MTC, it is still controversial. MTC patients without distant metastases have not been investigated to explore indications for adjuvant radiotherapy. This study aims to investigate the impact of radiotherapy on the survival of MTC patients without distant metastases.

Methods: Data of MTC patients without distant metastasis who underwent total thyroidectomy between 2010 and 2015 were obtained from the Surveillance, Epidemiology and End Results (SEER) database. Propensity score matching was performed to analyze the relationship between radiotherapy and cancerspecific survival (CSS).

Results: Seventy-four of 718 MTC patients without distant metastases received radiotherapy and underwent total thyroidectomy. A total of 148 patients were screened via propensity score matching analysis. Multivariate Cox regression indicated that factors including age, sex, radiotherapy and chemotherapy were independent predictors of CSS. Based on these factors, MTC patients without distant metastasis were classified into two risk groups using a nomogram and risk classification system. The C-index of the nomogram was 0.791 . The calibration curves showed good consistency of CSS between the actual observation and the nomogram prediction, and decision curve analysis (DCA) showed great clinical usefulness of the nomogram. The three-year CSS of the radiotherapy group was $85.3 \%$, and that of the surgery group was $95 \%$. Particularly, compared with the surgery group, the three-year CSS of subgroups of the radiotherapy group, including male patients and those aged $>48$ years, was decreased.

Conclusions: Radiotherapy results in worse CSS for MTC patients without distant metastases. To maximize benefits, decisions about individual radiotherapy should weigh its advantages and disadvantages.

Keywords: Medullary thyroid cancer (MTC); radiotherapy; prognosis; propensity score matching; The Surveillance Epidemiology and End Results database (SEER database)

Submitted Jul 05, 2021. Accepted for publication Sep 16, 2021.

doi: $10.21037 /$ tcr-21-1179

View this article at: https://dx.doi.org/10.21037/tcr-21-1179

\section{Introduction}

Medullary thyroid carcinoma (MTC) accounts for 2-4\% of all thyroid malignancies (1). Derived from parafollicular cells of the thyroid, MTC is a neuroendocrine tumor. The degree of tumor malignancy is higher than that of other thyroid cancers (2). About $80 \%$ of MTC occurs in sporadic form and $20 \%$ occurs in genetic form. And hereditary MTC may be predicted due to germline mutations of the RET protooncogene $(3,4)$. 
MTC currently has a poor prognosis and few clinical treatments, and it presents more advanced disease symptoms and a worse prognosis than well-differentiated thyroid carcinoma (5). On presentation, half of patients have local lesions, while $35 \%$ of patients extend to surrounding tissues and $13 \%$ of patients have distant metastases (6). The mainstream treatment of MTC mainly focuses on traditional surgical resection. According to the 2009 American Thyroid Association (ATA) guidelines, surgery is the preferred radical treatment of medullary thyroid cancer, including total thyroidectomy and central lymph node dissection. Lateral neck dissection is performed in cases of lymph node metastasis or an increased risk of nodules (7). Other adjuvant treatments for MTC include radiotherapy and chemotherapy (7). 2015 ATA guidelines also recommend that adjuvant radiotherapy should be considered in patients with incomplete resection or a high risk of local recurrence (8). MTC appears to be a radiosensitive tumor. Radiotherapy may be an effective adjunct to prevent local recurrence, and it may also provide long-lasting and continuous control of locally advanced or metastatic disease (or both) in certain MTC patients (9). Twenty-nine nonmetastatic locally advanced MTC patients who received radiotherapy after surgery were enrolled in a study in 2016. The 5 -year local recurrence-free survival rate was $79 \%$ (10). However, the impact of radiotherapy on survival is still controversial. When patients were divided into two groups with the same age and stage of illness; the survival rate of the surgery-only group (29 patients) was significantly higher than that of the tumor surgery with postoperative adjuvant external radiotherapy group (15 patients) (11). The 5 -year survival rate of surgery in the postoperative adjuvant external radiotherapy group was $97 \%$ among 37 MTC patients with a primary tumor infiltrating beyond the thyroid capsule, while that in the surgery alone group was only $62 \%$ (12). Most studies focus on MTC patients who have advanced disease symptoms or distant metastases, and there are no studies on the survival rate of MTC patients without distant metastasis.

In general, due to the lack of prospective studies with treatment randomization and the obstacles for conducting prospective studies, it is challenging to evaluate the benefits of radiotherapy in MTC patients without distant metastasis. As a retrospective study, sample size and follow-up time are vital factors for comparative analysis of validity. Therefore, the purpose of this study was to provide a comprehensive comparison of the survival outcomes among MTC patients using population-based data to compare the use of radiotherapy with no radiotherapy in the management of adjuvant therapy for MTC patients without distant metastasis.

We present the following article in accordance with the STROBE reporting checklist (available at https://dx.doi. org/10.21037/tcr-21-1179).

\section{Methods}

\section{Data source and study population}

The Surveillance Epidemiology and End Results (SEER) database is the authoritative source of cancer data in the U.S., and it includes 18 regional cancer registries that cover nearly $30 \%$ of the U.S. population, with data related to cancer-based sociodemographic factors, clinicopathological factors, stage at diagnosis, primary treatment, and follow-up survival (13).

We used SEER Stata software to extract individual data of patients treated with total thyroidectomy for MTC between 2010 and 2015 in the SEER database. The inclusion criteria for the current study were as follows: (I) diagnosis of MTC as the primary tumor with positive histological confirmation (ICD histology code: 8510); (II) underwent total thyroidectomy; (III) no distant metastasis (M0 stage); (IV) survival status and time were known; and (V) sociodemographic characteristics (age at diagnosis, sex, race, marital status), clinicopathological characteristics [tumor grade, tumor stage, tumor size, extrathyroidal extension (ETE) and tumor multifocality] and treatmentrelated information (surgical procedures, radiotherapy and chemotherapy) were available. Normally, the American Joint Commission on Cancer (AJCC) guidelines are used to categorize the TNM stage. Based on the CS tumor code, the tumor size was divided into three groups: " $<2 \mathrm{~cm}$ ", "2-4 cm" and " $>4 \mathrm{~cm}$ ". The exclusion criteria were as follows: (I) no specific treatment-related information; (II) unknown specific cause of death; (III) patients who lacked sociodemographic or clinicopathological data; (IV) carcinoma with distant metastasis (M1 stage); and (V) patients without total thyroidectomy.

\section{Propensity score matching}

This was a retrospective study but not a randomized controlled trial (RCT); therefore, selection bias was inevitable. Traditional 1:1 propensity score matching (PSM) was introduced to minimize the effect of bias, which 


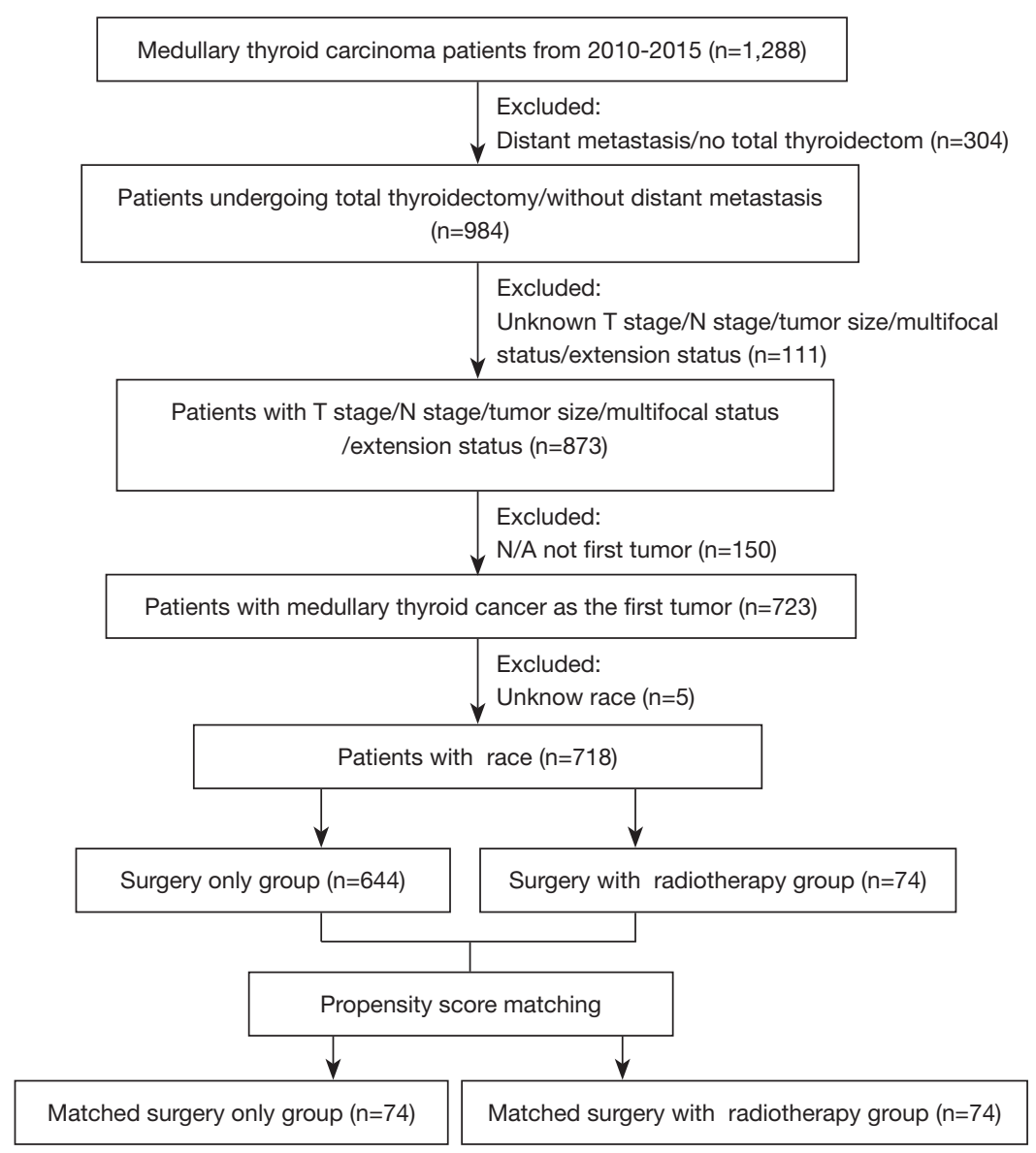

Figure 1 Flow diagram of the included medullary thyroid cancer patients.

might affect therapy decisions in MTC patients. PSM analysis consisted of 10 adjustment variables, including age, race, sex, tumor size, clinical $\mathrm{T}$ stage, $\mathrm{N}$ stage, TNM stage, ETE, tumor multifocality and chemotherapy, and achieved a similar randomization effect. After PSM analysis, no significant differences were observed in the above adjustment variables in the matched cohorts. The final study sample contained 148 patients (Figure 1).

\section{Statistical analysis}

Differences in sociodemographic, clinicopathological and treatment characteristics were compared by the chisquared test. Univariate and multivariate Cox survival regression analyses were conducted in the PSM-adjusted cohort to explore independent predictors associated with cancer-specific survival (CSS) in MTC patients without distant metastasis. Corresponding hazard ratios (HRs) along with $95 \%$ CIs are also presented. The nomogram was further established based on significant predictors in the multivariate analysis model to predict the probability of 3 -year and 5-year survival. Calibration curves were used to illustrate the relationship between the predicted and actual survival. Decision curve analysis (DCA) was also used to assess the prognostic capacity of the independent predictors. In addition, a risk classification system was established according to the total scores of each patient using a nomogram to divide all patients into two prognostic groups. Kaplan-Meier curves along with log-rank tests were applied to compare the CSS of patients in the two risk groups and to compare variables, including radiotherapy, sex, age and chemotherapy treatment of the primary tumor.

All statistical analyses were performed with SPSS version 20.0 (IBM Corporation, Armonk, NY, USA). The cutoff value of age was analyzed using the $\mathrm{X}$-tile program. The optimal age cutoff values for CSS determined in this study 

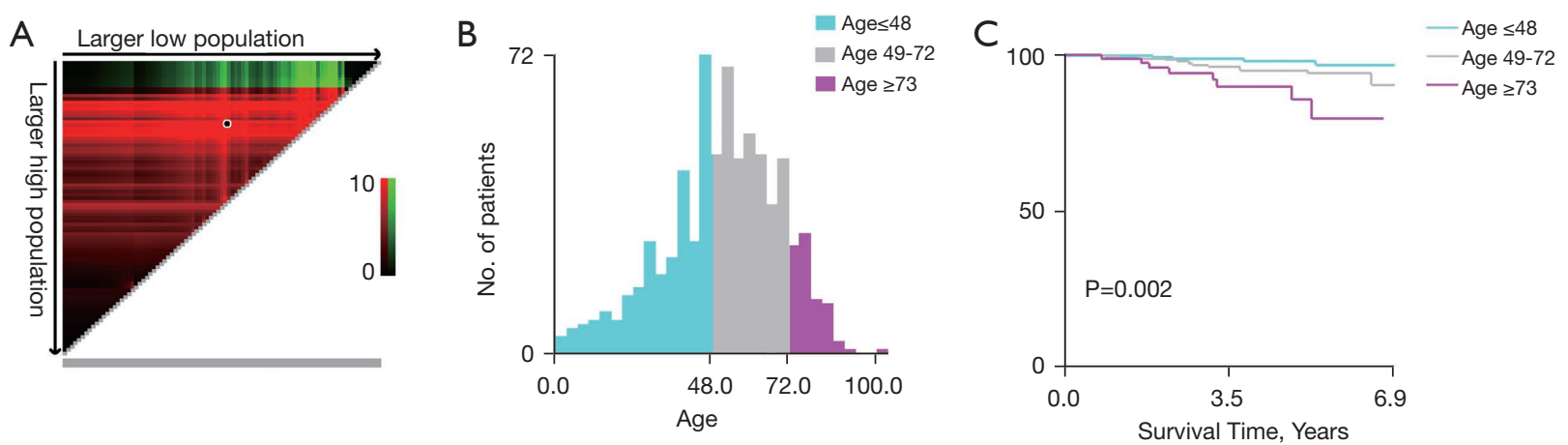

Figure 2 X-tile analysis of survival data from the SEER registry. The optimal cut-point highlighted by the black circle in the left panels (A) is shown on a histogram of the entire cohort (B) and a Kaplan-Meier plot (C). Figures show age divided at the optimal cut-point (48 and 72 , $\left.\chi^{2}=12.047, \mathrm{P}=0.002\right)$.

were 48 and 73 years (Figure 2). Survival curves were generated by GraphPad Prism 7.0 (GraphPad Software, San Diego, CA, USA). The nomogram and risk classification system were constructed with $\mathrm{R}$ studio software. PSM was performed using " $\mathrm{R}$ studio" together with the $\mathrm{R}$ packages "tableone" and "nonrandom". Variables with a $\mathrm{P}$ value $<0.1$ in the univariate Cox survival analysis were included in the multivariate Cox regression analysis, and a $\mathrm{P}$ value $<0.05$ in the other analyses was considered to be statistically significant.

\section{Results}

\section{Population characteristics}

A total of 718 MTC patients who underwent total thyroidectomy between 2010 and 2015 and had no distant metastasis were screened from the SEER database based on the inclusion criteria. Among these patients, $644(89.7 \%)$ underwent surgery alone, and 74 (10.3\%) received surgery with radiotherapy. The sociodemographic, clinicopathological and treatment characteristics of the patients are summarized in Table 1 . Overall, $85.8 \%$ of patients were Caucasian (white race), and $60.2 \%$ were female. Regarding tumor size, $55.4 \%$ of patients had tumors $\leq 2 \mathrm{~cm}$, while only $14.2 \%$ had tumors $>4 \mathrm{~cm}$. Among the 718 patients, $85.2 \%$ had no ETE, $32.7 \%$ had multifocal tumors, and only $1.9 \%$ received chemotherapy of the primary tumor. According to the cutoff points of age determined by $\mathrm{X}$-tile, patients were classified into three subgroups: $\leq 48$ years $(n=285), 49-72$ years $(n=348)$, and $\geq 73$ years $(\mathrm{n}=85)$. Before PSM analysis, patients in the surgery with radiotherapy cohort had a higher tumor grade (III/IV) $85.1 \%$ versus $35.1 \%(\mathrm{P}<0.001)$ relative to the surgery only cohort (Table 1). PSM was conducted on the basis of the propensity score, and 74 pairs of patients treated with surgery only and surgery plus radiotherapy were matched. No statistically significant difference was noted between baseline groups in PSM-adjusted cohorts (Table 1, Table S1).

\section{Predictors of CSS}

Univariable Cox regression analysis in the PSM-adjusted cohort was performed to assess the associations between covariates and CSS (Table 2, Table S2). The possible risk factors according to the univariable Cox regression analysis included group, older age, female sex, tumor size $>4 \mathrm{~cm}$, ETE and chemotherapy. Then, the multivariable Cox proportional hazards survival regression analysis revealed that patients who underwent surgery with radiotherapy had a higher likelihood of MTC mortality than those who underwent surgery only (HR: 4.701, 95\% CI: 1.435-15.403, $\mathrm{P}=0.011$ ) (Table 2). This was also the case for chemotherapy versus no chemotherapy (HR, 5.692; 95\% CI, 1.671-19.391, $\mathrm{P}=0.005$ ) (Table 2). In addition to treatment-related factors, age $\geq 73$ (HR: 16.302; 95\% CI: 3.139-84.657; $\mathrm{P}=0.001$ ) and female sex (HR: 0.188; 95\% CI: 0.051-0.690; $\mathrm{P}=0.012$ ) were also independent predictors of CSS in MTC patients without distant metastasis (Table 2). A nomogram for predicting the probability of 3 -year and 5-year survival was further established based on crucial independent hazard factors identified through multivariable Cox regression analyses (Figure 3). The CSS could be estimated 
Table 1 Baseline characteristics of total patients before and after propensity score matching

\begin{tabular}{|c|c|c|c|c|c|c|}
\hline Variables & \multicolumn{3}{|c|}{ Before matching } & \multicolumn{3}{|c|}{ After matching } \\
\hline Age (years) & & & 0.680 & & & 0.741 \\
\hline$\leq 48$ & 257 (39.9\%) & $28(37.8 \%)$ & & $25(33.8 \%)$ & $28(37.8 \%)$ & \\
\hline Race & & & 0.415 & & & 0.901 \\
\hline White & $549(85.2 \%)$ & 67 (90.5\%) & & 68 (91.9\%) & $67(90.5 \%)$ & \\
\hline Black & $46(7.1 \%)$ & $3(4.1 \%)$ & & $2(2.7 \%)$ & $3(4.1 \%)$ & \\
\hline Female & $400(62.1 \%)$ & $32(43.2 \%)$ & & $35(47.3 \%)$ & $32(43.2 \%)$ & \\
\hline Tumor size $(\mathrm{cm})$ & & & $<0.001$ & & & 0.976 \\
\hline$\leq 2$ & 377 (58.5\%) & $21(28.4 \%)$ & & $22(29.7 \%)$ & $21(28.4 \%)$ & \\
\hline $2-4$ & $185(28.7 \%)$ & $33(44.6 \%)$ & & $33(44.6 \%)$ & $33(44.6 \%)$ & \\
\hline$>4$ & 82 (12.7\%) & $20(27.0 \%)$ & & $19(25.7 \%)$ & $20(27.0 \%)$ & \\
\hline TNM stage & & & $<0.001$ & & & 0.821 \\
\hline $\mathrm{I} / \mathrm{II}$ & 418 (64.9\%) & $11(14.9 \%)$ & & $12(16.2 \%)$ & $11(14.9 \%)$ & \\
\hline No & $420(65.2 \%)$ & $13(17.6 \%)$ & & $13(17.6 \%)$ & $13(17.6 \%)$ & \\
\hline $\mathrm{N} 1$ & 224 (34.8\%) & $61(82.4 \%)$ & & $61(82.4 \%)$ & $61(82.4 \%)$ & \\
\hline ETE & & & $<0.001$ & & & 0.869 \\
\hline No & 577 (89.6\%) & 35 (47.3\%) & & $34(45.9 \%)$ & $35(47.3 \%)$ & \\
\hline Yes & 67 (10.4\%) & 39 (52.7\%) & & $40(54.1 \%)$ & $39(52.7 \%)$ & \\
\hline Multifocality & & & 0.011 & & & 0.869 \\
\hline Solitary tumor & $443(68.8 \%)$ & $40(50.1 \%)$ & & $41(55.4 \%)$ & $40(54.1 \%)$ & \\
\hline Multifocal tumor & 201 (31.2\%) & $34(45.9 \%)$ & & $33(44.6 \%)$ & $34(45.9 \%)$ & \\
\hline Chemotherapy & & & 0.002 & & & 0.754 \\
\hline No & 635 (98.6\%) & 69 (93.2\%) & & 68 (91.9\%) & 69 (93.2\%) & \\
\hline Yes & $9(1.4 \%)$ & $5(6.8 \%)$ & & $6(8.1 \%)$ & $5(6.8 \%)$ & \\
\hline
\end{tabular}

ETE, extrathyroidal extension; TNM, tumor node metastasis. 
Table 2 Cox proportional hazard regression analysis for variables associated with CSS

\begin{tabular}{|c|c|c|c|c|}
\hline Variables & \multicolumn{2}{|c|}{ Univariate analysis } & \multicolumn{2}{|c|}{ Multivariate analysis } \\
\hline \multicolumn{5}{|l|}{ Group } \\
\hline Surgery only & Reference & & Reference & \\
\hline Surgery with radiotherapy & $2.978(0.960-9.240)$ & 0.059 & $4.701(1.435-15.403)$ & 0.011 \\
\hline$\leq 48$ & Reference & & Reference & \\
\hline $49-72$ & $1.935(0.523-7.162)$ & 0.323 & $1.910(0.514-7.105)$ & 0.334 \\
\hline$\geq 73$ & $6.481(1.428-29.419)$ & 0.015 & $16.302(3.139-84.657)$ & 0.001 \\
\hline \multicolumn{5}{|l|}{ Race } \\
\hline \multicolumn{5}{|l|}{ Sex } \\
\hline Male & Reference & & Reference & \\
\hline Female & $0.252(0.072-0.887)$ & 0.032 & $0.188(0.051-0.690)$ & 0.012 \\
\hline \multicolumn{5}{|l|}{ Tumor size (cm) } \\
\hline$\leq 2$ & Reference & & & \\
\hline $2-4$ & $2.051(0.397-10.581)$ & 0.391 & & \\
\hline$>4$ & $6.523(1.406-30.269)$ & 0.017 & & \\
\hline \multicolumn{5}{|l|}{ TNM stage } \\
\hline \multicolumn{5}{|l|}{$\mathrm{N}$ stage } \\
\hline No & Reference & & & \\
\hline N1 & $1.530(0.348-6.737)$ & 0.574 & & \\
\hline \multicolumn{5}{|l|}{ ETE } \\
\hline No & Reference & & & \\
\hline Yes & 3.131 (1.006-9.745) & 0.049 & & \\
\hline \multicolumn{5}{|l|}{ Multifocality } \\
\hline Solitary tumor & Reference & & & \\
\hline Multifocal tumor & $1.664(0.619-4.474)$ & 0.313 & & \\
\hline \multicolumn{5}{|l|}{ Chemotherapy } \\
\hline No & Reference & & Reference & \\
\hline Yes & $3.547(1.141-11.021)$ & 0.029 & 5.692 (1.671-19.391) & 0.005 \\
\hline
\end{tabular}

CSS, cancer-specific survival; ETE, extrathyroidal extension; HR, hazard ratio. 


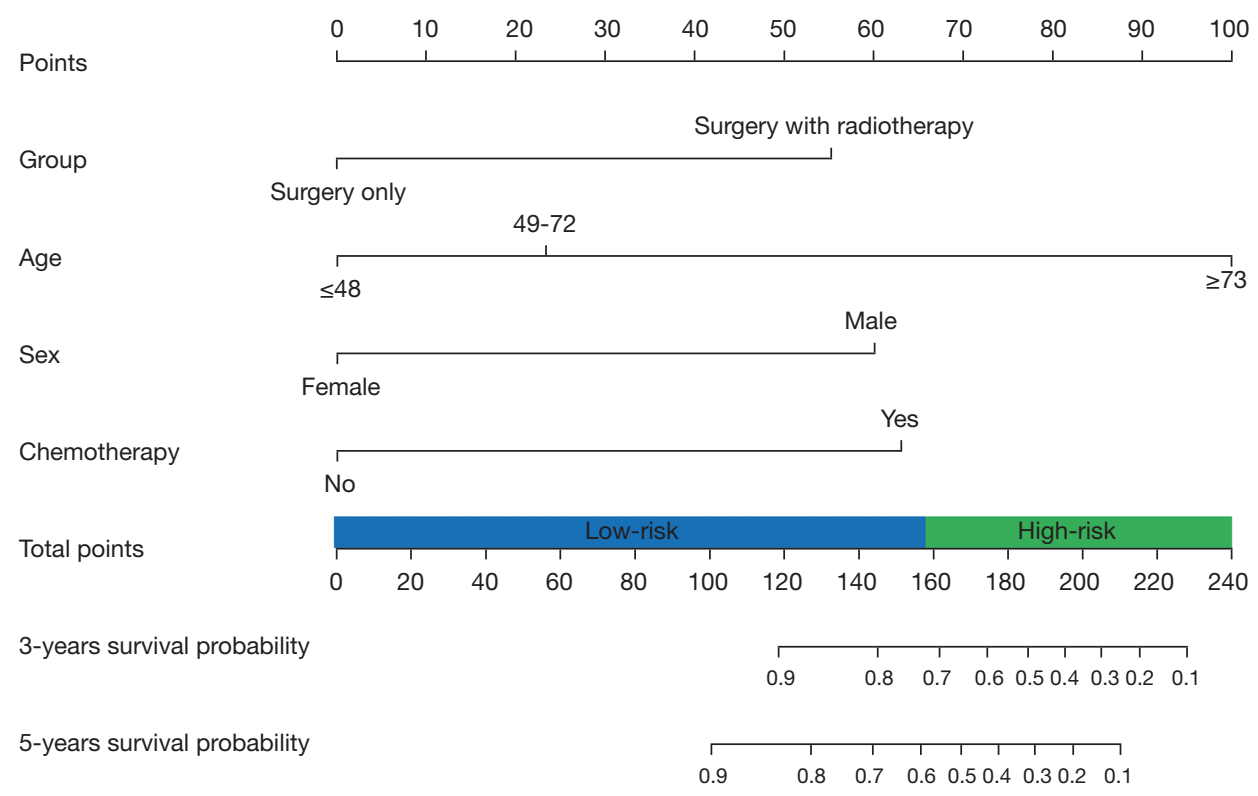

Figure 3 Nomogram and risk classification system of 3- and 5-year CSS for medullary thyroid cancer patients in the PSM-adjusted cohort. CSS, cancer-specific survival; PSM, propensity score matching.

with better predictive accuracy because our nomogram predicted survival precisely with a C-index of 0.791 , and the calibration curve also showed optimal agreement between the predicted probability of and observed 3- and 5-year CSS (Figure 4A,4B). Furthermore, DCA demonstrated that this predictive model could provide the best prediction of 3-year and 5-year CSS (Figure 5A,5B). Taken together, these results indicated that the nomogram, built with the above key independent hazard factors, not only was the best method for predicting 3-year and 5-year CSS for MTC patients without distant metastasis but also might help in clinical management.

\section{Risk classification system}

According to the total scores of each patient determined by the nomogram, a risk classification system for CSS was developed to classify all patients into two prognostic groups. Using this novel classification system, all patients were classified into low-risk $(116 / 148,78.38 \%$; score $0-157)$ and high-risk (32/148, 21.62\%; score 157-213) groups (Figure 3). The Kaplan-Meier curve demonstrated that CSS was accurately differentiated between the two groups by the risk classification system (Figure 6). For all patients, the median CSS times of patients in the low-risk and high-risk groups were 38.0 and 30.0 months, respectively.

\section{Matched cohort analysis and survival rate}

After PSM was performed to attenuate the bias of confounding factors and made the effects of radiotherapy more comparable, patients treated with surgery only had significantly higher CSS than those who underwent surgery with radiotherapy (Figure $7 A$ ). We further analyzed the effects of sex, age and chemotherapy on CSS in MTC patients who were treated with total thyroidectomy and had no distant metastasis. Within the PSM matched cohort, female patients were significantly superior to male patients $(\mathrm{CSS}-\mathrm{P}=0.007)$ in terms of MTC survival (Figure $7 B$ ). Moreover, as illustrated in Figure $7 C$, patients who were aged $\leq 48$ years had significantly higher CSS than those who were aged $\geq 73$ years or between 49 and 72 years $(\mathrm{P}=0.007)$ (Figure 7C). The 3-year CSS rates of the groups with and without chemotherapy were $94.4 \%$ and $57.7 \%$, respectively (Figure 7D).

When it comes to subgroups, the 3-year CSS rates of the surgery only group and surgery with radiotherapy group were $87.4 \%$ versus $65.1 \%$ for male patients $(\mathrm{P}=0.049)$ (Figure 8A). We used $\mathrm{X}$-tile to analyze the cutoff points for age $(\mathrm{P}=0.002)$. The 3 -year CSS rates of the surgery only group versus the surgery with radiotherapy group were $95 \%$ versus $72.2 \%$ among patients aged $49-72$ years $(\mathrm{P}=0.021)$ (Figure $8 B$ ) and $85.7 \%$ 

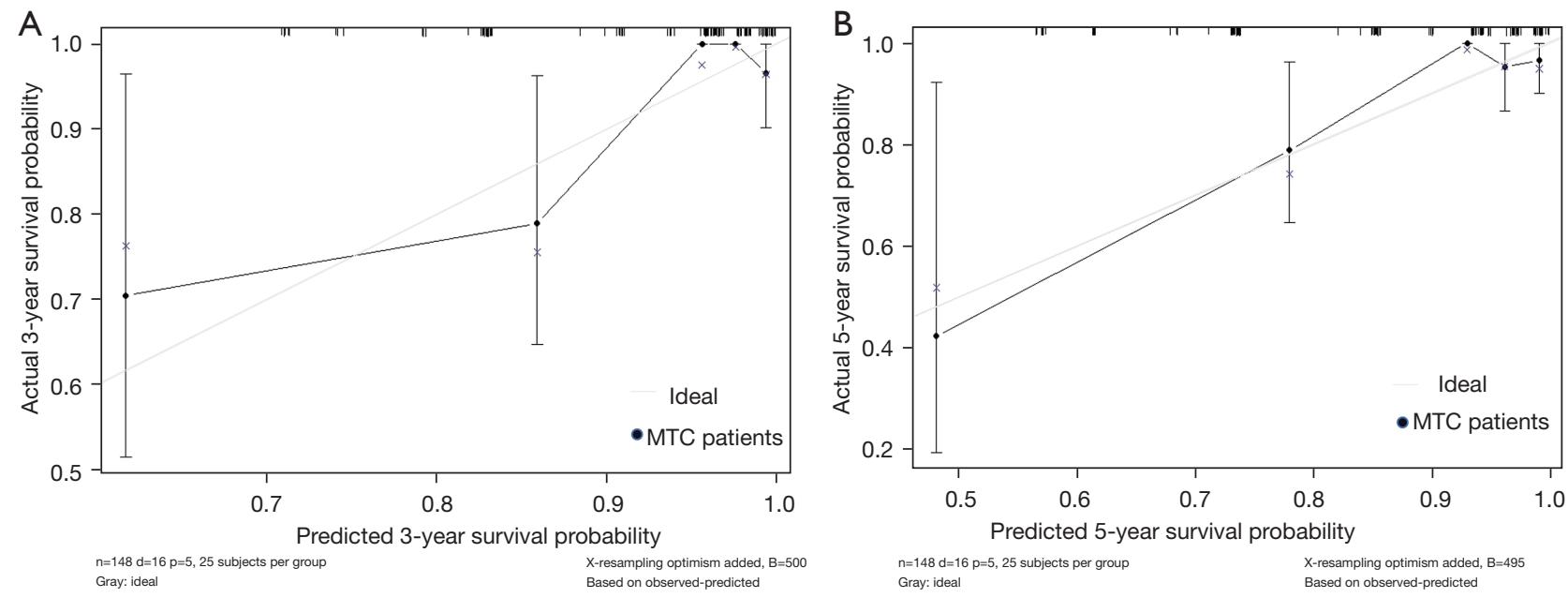

Figure 4 Calibration curves for predictions of CSS. (A) 3-year survival probability; (B) 5-year survival probability. Dashed line represents ideal prediction, and solid line represents observed nomogram. Vertical bars indicate $95 \%$ CIs, and crosses indicate bias-corrected estimates. CSS, cancer-specific survival.
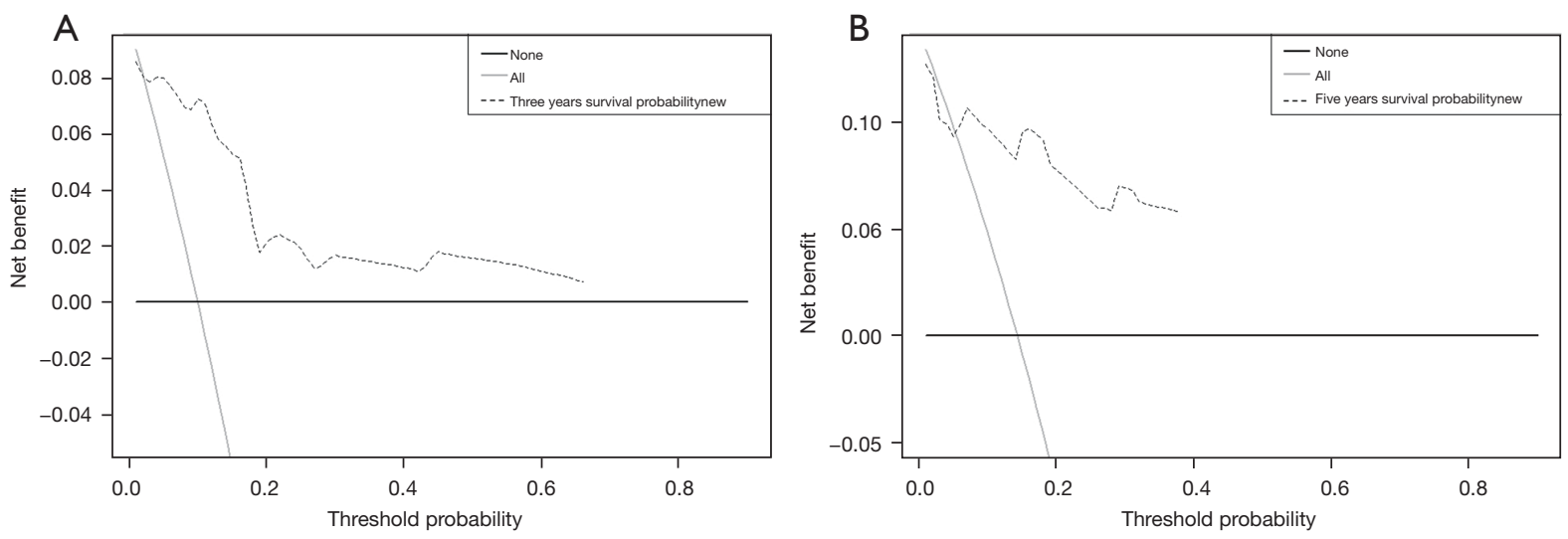

Figure 5 DCA for the survival nomogram. (A) DCA for 3-years survival probability; (B) DCA for 5-years survival probability. DCA, decision curve analysis.

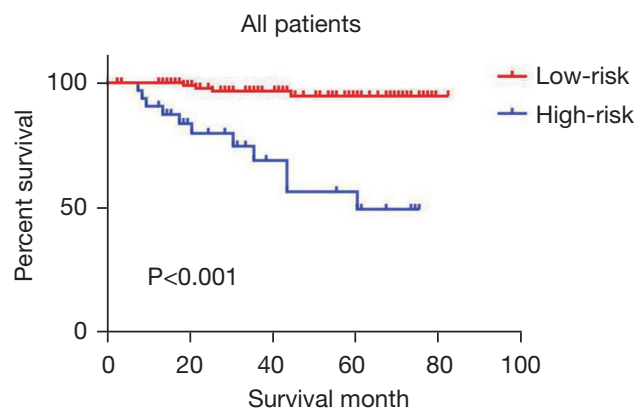

Figure 6 Kaplan-Meier curves of CSS for patients in the low-risk and high-risk groups. CSS, cancer-specific survival. versus $41.7 \%$ among patients aged $\geq 73$ years $(\mathrm{P}=0.030)$ (Figure $8 C$ ). Furthermore, the surgery only group had a more significant advantage with regard to CSS than the surgery with radiotherapy group. Similar results were also shown in patients who did not undergo chemotherapy (96.4\% versus $85.5 \%$; $\mathrm{P}=0.007$ ) (Figure $8 D$ ). Nevertheless, there was no significant difference in the CSS between the surgery only group and the surgery with radiotherapy group among female patients $(\mathrm{CSS}-\mathrm{P}=0.175)$, patients aged $\leq 48$ years $(\mathrm{CSS}-\mathrm{P}=0.873)$ and chemotherapy patients (CSS-P=0.798) (Figure 8E-8G). 

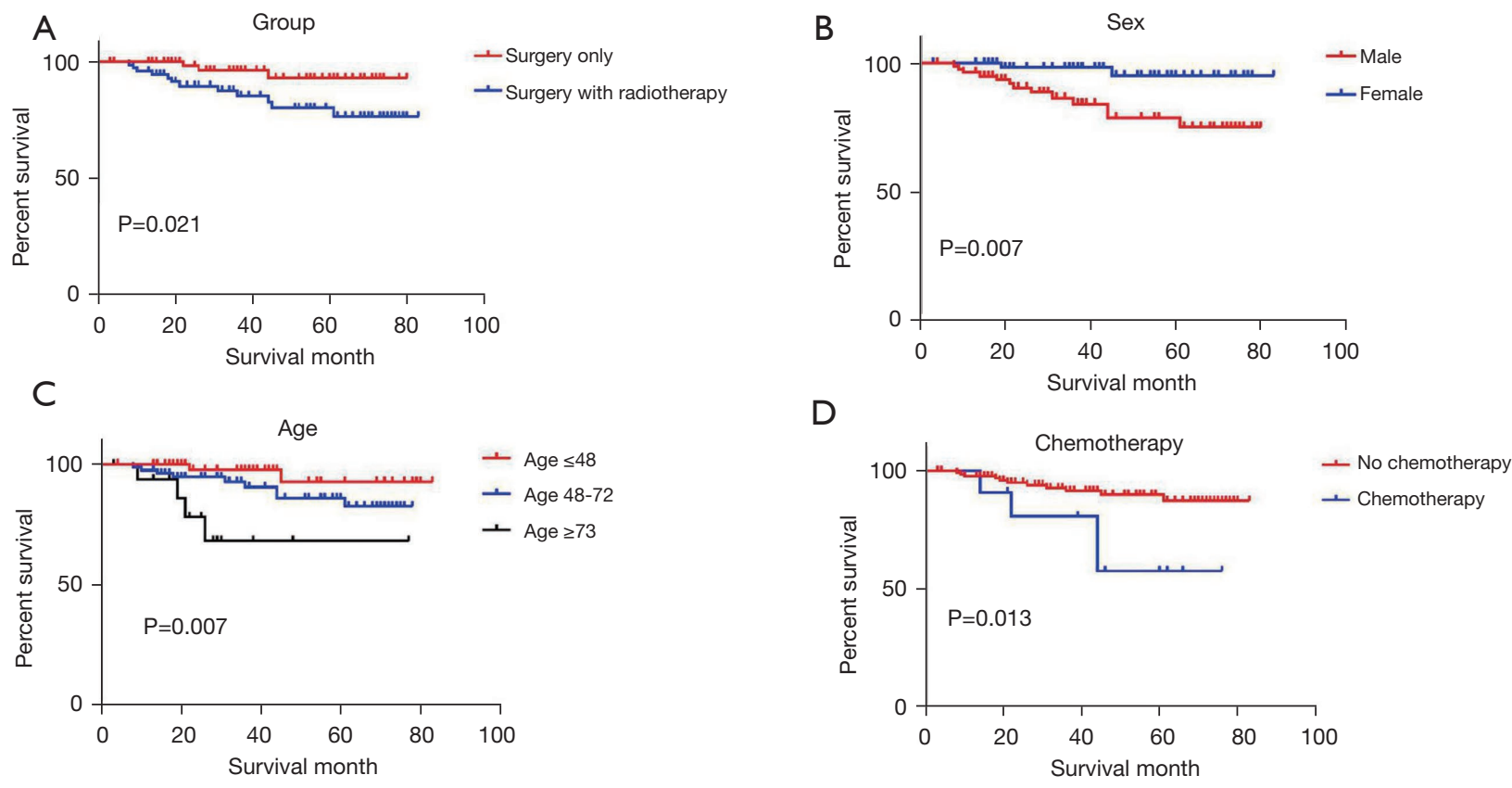

Figure 7 Kaplan-Meier curves survival analysis in the PSM-adjusted cohort. (A) CSS based on radiation exposure; (B) CSS based on sex; (C) CSS based on age; (D) CSS based on chemotherapy exposure. PSM, propensity score matching; CSS, cancer-specific survival.

\section{Discussion}

This study aims to provide a prognostic model and risk classification system to determine whether MTC patients without distant metastasis need to be recommended for radiotherapy and to resolve the controversy surrounding radiotherapy. At present, treatment of different stages of MTC with the same radiotherapy regimens may lead to different cost effects, such as quality of life, survival rate and radiation toxicity. Many studies have focused only on evaluating the effects of radiotherapy in MTC, but the M0 and $M 1$ stages were not discussed individually.

There was no significant difference in recurrence-free survival or overall survival (OS) between patients who received external beam radiotherapy (EBRT) and patients who only received surgery in a study including 539 MTC patients. Nevertheless, patients who received surgery had less overall discomfort than patients who received EBRT (14). Radiotherapy treatment in MTC patients is still controversial because research shows that radiotherapy can be effective for local control, but there is a lack of convincing evidence that postoperative radiotherapy affects the survival of MTC patients (15). EBRT has an adverse effect on OS $(16,17)$, but has no effect on other outcomes (18). Interestingly, a significant benefit for
OS was revealed in a retrospective study of 91 clinical cases of MTC in which patients were followed up for an average of 6 years (19). Radiotherapy can provide clinically meaningful benefits by protecting the airway, esophagus, and peripheral neurovascular structures to improve quality of life. Therefore, local radiotherapy treatment for local disease control is recommended for patients with a high risk of local recurrence, advanced disease, or distant metastases due to obvious local regional progression symptoms, but it may not provide survival benefits. However, a separate assessment of the relevant role of radiotherapy in MTC patients without distant metastasis should be conducted. To maintain the expected survival benefits, the decision to recommend radiotherapy should be further explored to balance the potential toxicity triggered by radiotherapy and local treatment.

This study used population-based data to select M0 stage and total thyroidectomy MTC patients to clarify the impact of radiotherapy on the survival of MTC patients without distant metastasis. This study demonstrates that the addition of radiotherapy to surgical treatment leads to worse CSS, particularly in men and patients aged $>48$ years, according to propensity score matching. However, factors including female sex, age $\leq 48$ years, and lack of adjuvant radiotherapy all resulted in higher survival rates. The 3-year survival 

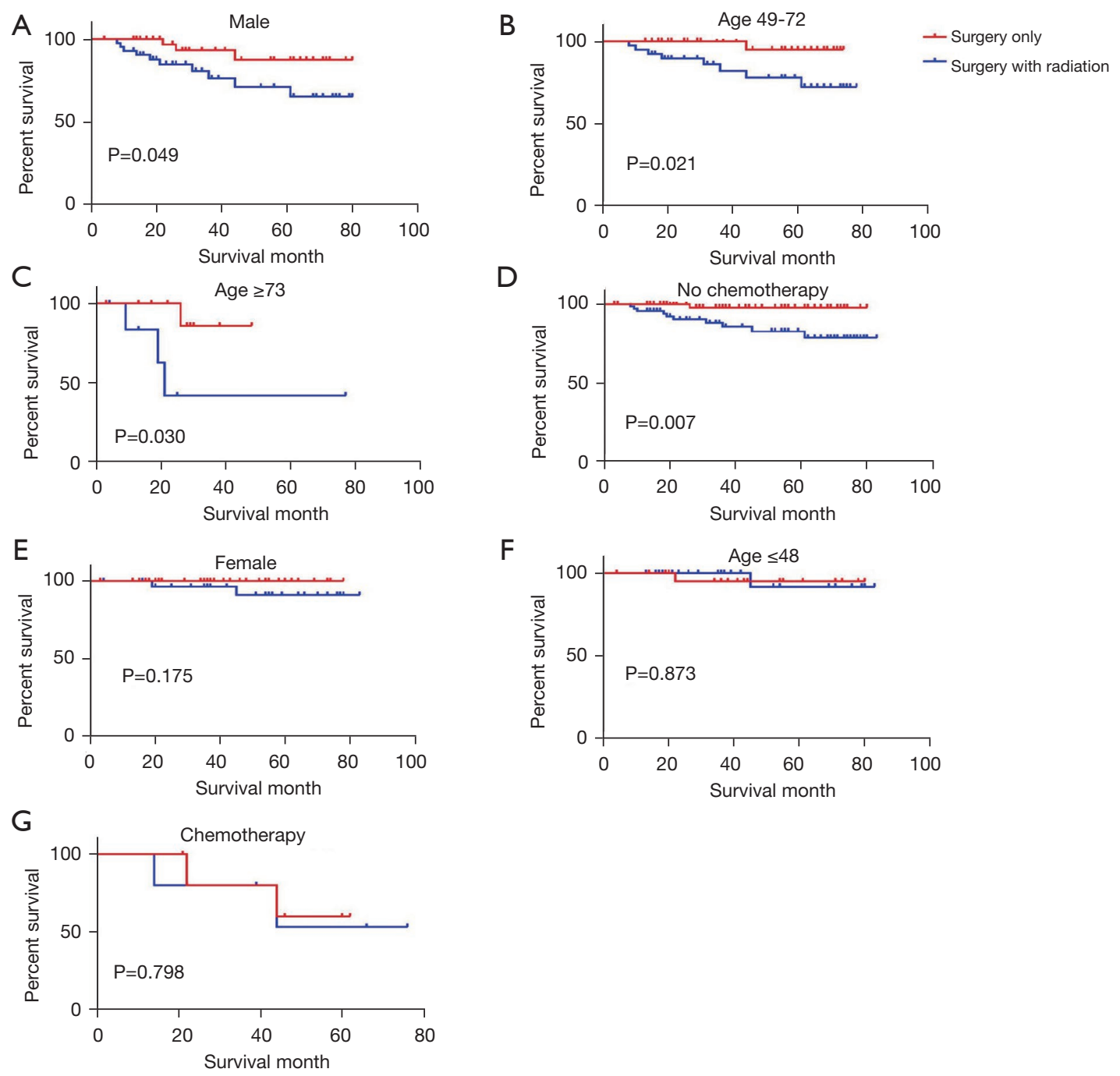

Figure 8 Kaplan-Meier curves of CSS comparing between surgery only group and surgery with radiotherapy group. (A) Males; (B) females; (C) age $\leq 48$; (D) age 49-72; (E) age $\geq 73$; (F) no chemotherapy; (G) chemotherapy. CSS, cancer-specific survival.

rate of the surgery group was $95 \%$, and the 3 -year survival rate of the surgery combined with radiotherapy group was $85.3 \%(\mathrm{P}=0.021)$ (Figure $7 A)$, which was consistent with the conclusions of other scholars (11). The prognosis of patients receiving radiotherapy in this study was poor. Since the study subjects were patients without distant metastases, the risk of tumor recurrence was low, and radiotherapy may do more harm than good in this population. MTC patients without distant metastasis may not be the ideal target for radiotherapy. Hence, the potential benefits of radiotherapy for local control should be fully weighed against the possibility of acute and late toxicity. The decision to recommend radiotherapy in these patients must vary among patients. The impact of radiotherapy on the prognosis of M0 stage MTC patients should be examined in a large-scale prospective study.

In addition, this study revealed that chemotherapy significantly reduced the survival rate of MTC patients without distant metastasis, which is similar to the conclusions of previous studies (20) (Figure 7D). Moreover, the multivariate Cox regression analysis and nomogram both showed clinically significant differences in sex and age in MTC patients. Male sex and age $>48$ years old are factors affecting poor prognosis. Multiple studies have found that age is one of the main determinants of CSS in MTC (21). Age has been identified as an independent risk 
factor, suggesting that the survival rate of elderly patients is low $(22,23)$. Most studies revealed a lower survival rate among male MTC patients (24-27). Considering the high prevalence of thyroid nodules in men $(80 \%$ in men compared to $56 \%$ in women), the association with thyroid nodules seems to be the basis for the poor prognosis. The size of the primary tumor and extracellular invasion of the tumor are independent prognostic factors (28). This study also found that the size of the primary tumor and extracapsular invasion both affected the prognosis in the univariate analysis but did not affect the prognosis in the multivariate analysis. This may be linked to MTC patients without distant metastasis, who generally have mild local symptoms. The size of the primary tumor and extracapsular invasion were not the main factors affecting the prognosis. Cervical lymph node metastasis was reported as an independent factor affecting the prognosis of MTC patients (29), while another study (30) did not demonstrate that lymph node metastasis significantly affects the prognosis of patients, which is consistent with our study. In this study, M0 stage patients were examined, and the impact of the $M$ stage on prognosis was avoided. Because factors including the size of the primary tumor and cervical lymph node metastasis did not affect the prognosis in our study, this may explain why the TNM stage was not a statistically significant factor for the survival rate and prognosis.

As a retrospective analysis, this study has the following shortcomings. First, the collection and recording of baseline data and the inclusion and selection of data may be biased, resulting in deviations between the overall status of the included patients and the original overall status. Second, propensity score matching analysis cannot explain unmeasured variables. This study did not include other indicators that may affect the prognosis of medullary thyroid cancer, such as smoking, preoperative calcitonin level and surgical margin information. The SEER database does not provide specific information about radiotherapy, including the total dose, time relative to surgery and technique. Additional, this article focuses on the survival rate and does not conduct an in-depth investigation of local control because the SEER database does not collect information about recurrence.

\section{Conclusions}

This is the first study to explore the role of radiotherapy in the initial management of M0 stage MTC patients via a nomogram and risk classification system. This is also the first study to reveal through propensity score matching analysis that radiotherapy is associated with a poor prognosis for $\mathrm{M} 0$ stage patients and significantly reduces the survival rate. Although studies have shown that radiotherapy is beneficial to the local control of high-risk groups since 1996 (31), the decision to recommend radiotherapy for M0 stage patients should be carefully considered, and the advantages and disadvantages of radiotherapy should be comprehensively evaluated for each patient. This decisionmaking process will greatly benefit from prospective research because it more robustly incorporates variables related to tumor, surgical, and radiotherapy characteristics.

\section{Acknowledgments}

Funding: This work was supported by the National Natural Science Foundation of China (Grant No. 81770835) and the Key-Area Research and Development Program of Guangdong Province (Grant No. 2019B020230001).

\section{Footnote}

Reporting Checklist: The authors have completed the STROBE reporting checklist. Available at https://dx.doi. org/10.21037/tcr-21-1179

Data Sharing Statement: Available at https://dx.doi. org/10.21037/tcr-21-1179

Peer Review File: Available at https://dx.doi.org/10.21037/ tcr-21-1179

Conflicts of Interest: All authors have completed the ICMJE uniform disclosure form (available at https://dx.doi. org/10.21037/tcr-21-1179). The authors have no conflicts of interest to declare.

Ethical Statement: The authors are accountable for all aspects of the work in ensuring that questions related to the accuracy or integrity of any part of the work are appropriately investigated and resolved. The study was conducted in accordance with the Declaration of Helsinki (as revised in 2013).

Open Access Statement: This is an Open Access article distributed in accordance with the Creative Commons Attribution-NonCommercial-NoDerivs 4.0 International License (CC BY-NC-ND 4.0), which permits the non- 
commercial replication and distribution of the article with the strict proviso that no changes or edits are made and the original work is properly cited (including links to both the formal publication through the relevant DOI and the license). See: https://creativecommons.org/licenses/by-nc-nd/4.0/.

\section{References}

1. Ceolin L, Duval M, Benini AF, et al. Medullary thyroid carcinoma beyond surgery: advances, challenges, and perspectives. Endocr Relat Cancer 2019;26:R499-518.

2. Ganeshan D, Paulson E, Duran C, et al. Current update on medullary thyroid carcinoma. AJR Am J Roentgenol 2013;201:W867-76.

3. Leimbach RD, Hoang TD, Shakir MKM. Diagnostic Challenges of Medullary Thyroid Carcinoma. Oncology 2021;99:422-32.

4. Jozaghi Y, Zafereo M, Williams MD, et al. Neoadjuvant selpercatinib for advanced medullary thyroid cancer. Head Neck 2021;43:E7-12.

5. Oczko-Wojciechowska M, Czarniecka A, Gawlik T, et al. Current status of the prognostic molecular markers in medullary thyroid carcinoma. Endocr Connect 2020;9:R251-63.

6. Kushchayev SV, Kushchayeva YS, Tella SH, et al. Medullary Thyroid Carcinoma: An Update on Imaging. J Thyroid Res 2019;2019:1893047.

7. Panigrahi B, Roman SA, Sosa JA. Medullary thyroid cancer: are practice patterns in the United States discordant from American Thyroid Association guidelines? Ann Surg Oncol 2010;17:1490-8.

8. Wells SA Jr, Asa SL, Dralle H, et al. Revised American Thyroid Association guidelines for the management of medullary thyroid carcinoma. Thyroid 2015;25:567-610.

9. Call JA, Caudill JS, McIver B, et al. A role for radiotherapy in the management of advanced medullary thyroid carcinoma: the mayo clinic experience. Rare Tumors 2013;5:e37.

10. Compagnon F, Zerdoud S, Rives M, et al. Postoperative external beam radiotherapy for medullary thyroid carcinoma with high risk of locoregional relapse. Cancer Radiother 2016;20:362-9.

11. Samaan NA, Schultz PN, Hickey RC. Medullary thyroid carcinoma: prognosis of familial versus sporadic disease and the role of radiotherapy. J Clin Endocrinol Metab 1988;67:801-5.

12. Jensen MH, Davis RK, Derrick L. Thyroid cancer: a computer-assisted review of 5287 cases. Otolaryngol Head
Neck Surg 1990;102:51-65.

13. Qi L, Ren X, Liu Z, et al. Predictors and Survival of Patients with Osteosarcoma After Limb Salvage versus Amputation: A Population-Based Analysis with Propensity Score Matching. World J Surg 2020;44:2201-10.

14. Tubiana M, Haddad E, Schlumberger M, et al. External radiotherapy in thyroid cancers. Cancer 1985;55:2062-71.

15. Rowell NP. The role of external beam radiotherapy in the management of medullary carcinoma of the thyroid: A systematic review. Radiother Oncol 2019;136:113-20.

16. Dequanter D, Lothaire P. Medullary thyroid cancer: surgical results and prognostic factors. Rev Med Liege 2010;65:450-2.

17. Brierley J, Tsang R, Simpson WJ, et al. Medullary thyroid cancer: analyses of survival and prognostic factors and the role of radiation therapy in local control. Thyroid 1996;6:305-10.

18. Martinez SR, Beal SH, Chen A, et al. Adjuvant external beam radiation for medullary thyroid carcinoma. J Surg Oncol 2010;102:175-8.

19. Esik O, Tusnády G, Trón L, et al. Markov model-based estimation of individual survival probability for medullary thyroid cancer patients. Pathol Oncol Res 2002;8:93-104

20. Fialkowski EA, Moley JF. Current approaches to medullary thyroid carcinoma, sporadic and familial. J Surg Oncol 2006;94:737-47.

21. Shah S, Boucai L. Effect of Age on Response to Therapy and Mortality in Patients With Thyroid Cancer at High Risk of Recurrence. J Clin Endocrinol Metab 2018;103:689-97.

22. Shen W, Sakamoto N, Yang L. Cancer-specific mortality and competing mortality in patients with head and neck squamous cell carcinoma: a competing risk analysis. Ann Surg Oncol 2015;22:264-71.

23. Skillington SA, Kallogjeri D, Lewis JS Jr, et al. Prognostic Importance of Comorbidity and the Association Between Comorbidity and p16 in Oropharyngeal Squamous Cell Carcinoma. JAMA Otolaryngol Head Neck Surg 2016;142:568-75.

24. Raue F, Kotzerke J, Reinwein D, et al. Prognostic factors in medullary thyroid carcinoma: evaluation of 741 patients from the German Medullary Thyroid Carcinoma Register. Clin Investig 1993;71:7-12.

25. Saad MF, Ordonez NG, Rashid RK, et al. Medullary carcinoma of the thyroid. A study of the clinical features and prognostic factors in 161 patients. Medicine (Baltimore) 1984;63:319-42.

26. Gharib H, McConahey WM, Tiegs RD, et al. Medullary 
thyroid carcinoma: clinicopathologic features and longterm follow-up of 65 patients treated during 1946 through 1970. Mayo Clin Proc 1992;67:934-40.

27. Russell CF, Van Heerden JA, Sizemore GW, et al. The surgical management of medullary thyroid carcinoma. Ann Surg 1983;197:42-8.

28. Kazaure HS, Roman SA, Sosa JA. Medullary thyroid microcarcinoma: a population-level analysis of 310 patients. Cancer 2012;118:620-7.

29. Siironen P, Hagström J, Mäenpää HO, et al. Lymph

Cite this article as: Huang S, Zhong J, Zhang Z, Chen R, Li J, Sun J, Chen H. Prognosis of radiotherapy in medullary thyroid carcinoma patients without distant metastasis. Transl Cancer Res 2021;10(11):4714-4726. doi: 10.21037/tcr-21-1179 node metastases and elevated postoperative calcitonin: Predictors of poor survival in medullary thyroid carcinoma. Acta Oncol 2016;5 5:357-64.

30. Verbeek HH, Meijer JA, Zandee WT, et al. Fewer cancer reoperations for medullary thyroid cancer after initial surgery according to ATA guidelines. Ann Surg Oncol 2015;22:1207-13.

31. VFife KM, Bower M, Harmer CL. Medullary thyroid cancer: the role of radiotherapy in local control. Eur J Surg Oncol 1996;22:588-91. 
Supplementary

Table S1 Chi-square analysis on the degree of lymph node metastasis involvement after propensity score matching

\begin{tabular}{lcc}
\hline Variables & \multicolumn{2}{c}{ After matching } \\
\cline { 2 - 3 } & & Surgery with radiotherapy $(\mathrm{n}=74)$ \\
\hline Lymph node group & $0(0.0 \%)$ & $2(2.7 \%)$ \\
NA & $13(17.6 \%)$ & $13(17.6 \%)$ \\
0 & $32(43.2 \%)$ & $30(40.5 \%)$ \\
$1-10$ & $29(39.2 \%)$ & $29(39.2 \%)$ \\
\hline
\end{tabular}

Table S2 Cox proportional hazard regression analysis for the degree of lymph node metastasis involvement associated with CSS

\begin{tabular}{lcc}
\hline \multirow{2}{*}{ Variables } & \multicolumn{2}{c}{ Univariate analysis } \\
\cline { 2 - 3 } Lymph node group & $\mathrm{HR}(95 \mathrm{Cl})$ & $\mathrm{P}$ \\
NA & Reference & \\
0 & $0.161(0.015-1.793)$ & 0.138 \\
$1-10$ & $0.170(0.020-1.463)$ & 0.107 \\
$\geq 10$ & $0.305(0.038-2.452)$ & 0.264 \\
\hline
\end{tabular}

CSS, cancer-specific survival; HR, hazard ratio. 